\title{
The Role of Imaging in the Preoperative Assessment of Patients with Nasal Obstruction and Septal Deviation- A Retrospective Cohort Study
}

\author{
Narin Nard Carmel-Neiderman ${ }^{1}$ (1) Ahmad Safadi $^{1}$ Anat Wengier ${ }^{1}$ Tomer Ziv-Baran ${ }^{2}$ \\ Anton Warshavsky ${ }^{1}$ Barak Ringel $^{1} \quad$ Gilad Horowitz $^{1}$ Dan M. Fliss ${ }^{1}$ Avraham Abergel $^{1}$ \\ ${ }^{1}$ Department of Otolaryngology Head and Neck Surgery and \\ Maxillofacial Surgery, Tel-Aviv Sourasky Medical Center, Sackler \\ School of Medicine, Tel-Aviv University, Tel-Aviv, Israel \\ 2 Department of Epidemiology and Preventive Medicine, School of \\ Public Health, Sackler Faculty of Medicine, Tel-Aviv University, \\ Tel Aviv, Israel \\ Int Arch Otorhinolaryngol 2021;25(2):e242-e248.

\begin{abstract}
Address for correspondence Avraham Abergel, MD, Department of Otolaryngology, Head and Neck Surgery and Maxillofacial Surgery, Tel-Aviv Sourasky Medical Center, 6 Weizman Street, Tel-Aviv, 6423906, Israel (e-mail: Avrahamab@tlvmc.gov.il).
\end{abstract}

\begin{abstract}
Keywords

- nasal obstruction

- computed tomography

- septal deviation

Introduction Isolated nasal obstruction (INO) is a common complaint of multiple etiology. The preoperative evaluation of patients presenting with nasal obstruction and deviated nasal septum (DNS) does not typically include imaging. The benefits of performing computed tomography (CT) in the preoperative setting are inconclusive. Objective Assessing the contribution of preoperative $\mathrm{CT}$ to the surgical treatment of non-sinusitis patients presenting with INO and DNS.

Methods A retrospective cohort study on patients referred to surgery for nasal obstruction due to DNS or turbinate hypertrophy between 2006 and 2015. Data was retrieved from patients' medical charts. The $\mathrm{CT}$ scans and clinical data were reassessed by a second surgeon blinded to the patients' clinical course.

Results Seventy of the 843 patients (8.06\%) who underwent endoscopic sinonasal procedures during the study period had presented with INO and met the inclusion criteria. Thirty-eight (55.88\%) of them underwent CT scans during their preoperative assessment. Modification of the initial preoperative planning based on the radiological findings was required in 32 cases (84.2\%). When reassessed by a second blinded surgeon, $58 \%$ of cases required surgical modification rather than classical submucosal resection of nasal septum and turbinate reduction $(P=0.048)$.

Conclusion Computed tomography was found beneficial in the preoperative planning for patients with INO. The original surgical plan based upon physical examination findings was modified based on radiological findings in $84.2 \%$ of the patients.
\end{abstract}

received

September 1, 2019

accepted

April 18, 2020

published online

June 23, 2020
DOI https://doi.org/ 10.1055/s-0040-1712933. ISSN 1809-9777.

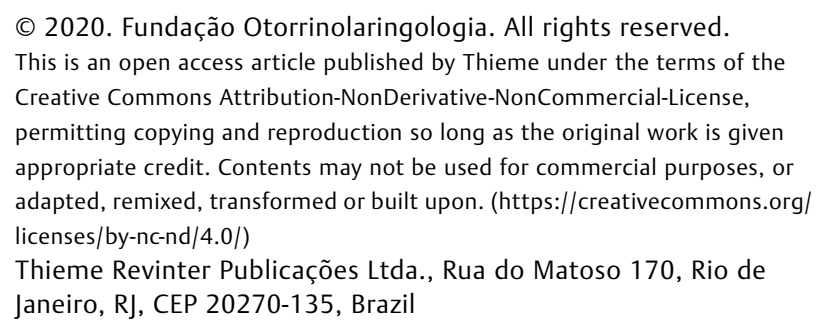




\section{Introduction}

Symptomatic nasal obstruction is a common complaint ${ }^{1}$. It may be attributed to several etiologies, such as severe septal deviation, mucosal congestion, turbinate hypertrophy, chronic rhinosinusitis, nasal polyps, tumors, and adenoid hypertrophy. Septoplasty and turbinate reduction are the common surgeries for non-sinusitis patients with isolated symptomatic nasal obstruction (INO). Preoperative assessment of patients presenting with isolated nasal airway obstruction might present a clinical challenge. In current practice, the decision to proceed to surgery and the extent of surgery in these patients is determined by clinical features, based on medical history and assessment of the nasal cavity on physical examination (including anterior rhinoscopy and fiber optic endoscopy). Recent review of long-term results of septoplasty concluded that it appears to be a far from perfect treatment for nasal obstruction due to septal deviation. This emphasizes the need for studies that will help define prognostic factors for nasal obstruction surgical treatment. ${ }^{1-3}$ Computed tomography (CT) is widely used in the preoperative assessment of patients with chronic sinusitis. It is essential for the surgical planning in these patients.

The preoperative evaluation of patients presenting with nasal obstruction and deviated nasal septum does not routinely include imaging studies; performing a CT scan as part of the surgical assessment and planning is not currently supported by consensus guidelines. ${ }^{4}$ Therefore, referral to preoperative CT studies is mainly based on clinical judgment. The benefits of performing CT in the preoperative setting of nasal obstruction and deviated nasal septum are unclear, and the relevant literature is scarce. A recent study by Karatas et al ${ }^{5}$ demonstrated the possible contribution of CT to the evaluation of other sinonasal anatomic variants that may contribute to the understanding of patients' sinonasal complaints and aid in surgical planning. ${ }^{6}$ However, while CT studies may provide additional information on the status of the nasal septum and the nasal cavity, the interpretation of this information is not straight-forward, mainly due to the high frequency of anatomical variations, even in the healthy asymptomatic population ${ }^{5}$.

In our outpatient clinic, we encountered patients who were referred to septoplasty and inferior turbinate reduction due to symptomatic INO who had performed a CT scan prior to their visit. It was our observation that the imaging performed led to a better understanding of the patients' condition and affected the surgical plan.

The objective of our study was to investigate the role of CT imaging studies in the preoperative assessment of patients presenting with INO and septal deviation.

\section{Materials and Methods}

Approval for conducting this study and for its design was obtained from the medical center's Institutional Review Board (0364-16-TLV).

\section{Patient Selection and Evaluation}

The present retrospective cohort study was conducted on patients undergoing sinus surgery in a tertiary medical center between 2006 and 2015. Adult (age ${ }^{3} 18$ years) patients who underwent endoscopic sinus surgery (ESS), septoplasty or submucosal resection of septum (SMR), and/or inferior turbinate reduction were considered for inclusion in the study, and their electronic medical records were reviewed.

We included patients who were referred to our rhinology clinic for surgical treatment due to INO and septal deviation. Patients whose major complaint was impaired nasal breathing with anatomical obstruction on clinical evaluation (i.e, septal deviation, turbinate hypertrophy) without inflammatory findings on physical examination were defined as suffering from INO. When we felt that the physical examination alone did not lead to adequate preoperative understanding of obstruction sites, a preoperative CT scan was performed. Patients with a history of sinus surgery, diagnosis of chronic sinusitis with or without polyposis, clinical symptoms or findings in physical examination typical of sinusitis, according to the American Academy of Otolaryngology Head and Neck Surgery Foundation, ${ }^{7}$ were excluded from the study. Also excluded were patients with available sinus $\mathrm{CT}$ scans prior to initial intake due to multiple causes.

Data retrieved included demographics (age, gender, allergic symptoms and allergy confirmed by skin tests), presenting symptoms (INO), clinical findings on physical examination (septal deviation, middle meatus abnormalities, pale edematous mucosa with appearance typical of allergy, inferior turbinate hypertrophy),imaging findings detected on CT scan (concha bullosa, mucosal thickening, inferior turbinate hypertrophy, evidence of sinus mucosal disease, osteomeatal complex [OMC] obstruction, and septal deviation) and operative course.

To objectively evaluate the added contribution of CT imaging for the surgeon, CT scans of 20 patients who had undergone imaging studies as part of their preoperative work-up were reviewed by a second blinded expert rhinologist, who evaluated the contribution of the CT scan to the choice of surgical approach (traditional SMR and turbinate reduction or whether functional endoscopic sinus surgery [FESS] or partial middle turbinectomy should be considered). The blinded expert only reviewed the patients' medical history, the documented physical examination in the outpatient clinic visit and the imaging studies and was unfamiliar with the original surgical plan and the selected surgical intervention.

\section{Statistical Analysis}

The statistical analysis was performed using SPSS Statistics for Windows, Version 24.0. (IBM Corp., Armonk, NY, USA). All statistical tests were two-tailed, and a $p$-value $<0.05$ was considered significant. Categorical variants were described as frequency and percentage. Continuous variables were evaluated for normal distribution and described as median and interquartile range or mean and standard deviation. Continuous variables were compared using the Mann- 


\section{3 patients underwent endoscopic sinus procedures (2006-2015)}

\section{2 (19.2\%) Initially presented with isolated nasal obstruction}

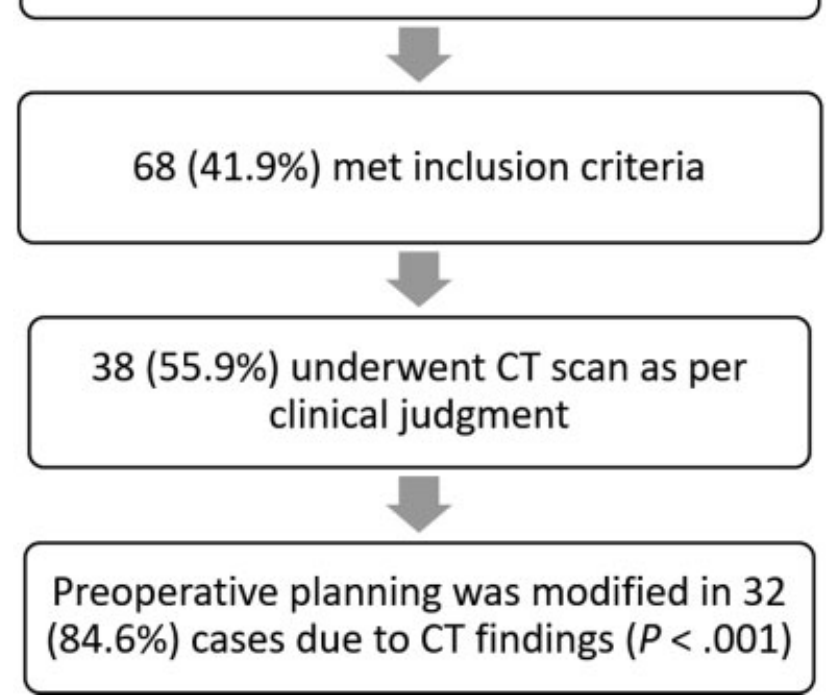

Fig. 1 Flowchart of patient course throughout the study.

Whitney test, and categorical variables were compared by the Chi-square test or Fisher exact test. A one-sample binomial test was performed to compare the independent blinded surgeon assessments of $\mathrm{CT}$ benefit to absolute value to detect significant surgeon-independent benefits.

\section{Results}

Of the 843 patients who underwent endoscopic sinonasal procedures between 2006 and 2015, a total of 68 patients met the inclusion criteria, and their medical records were reviewed by an expert rhinologist ( $\mathbf{- F i g . 1}$ ). The mean age of the patient cohort was $29.65 \pm 12.12$ years, with male predominance, 50 (73.5\%) patients were male ( - Table 1$)$. Twenty-one $(30.9 \%)$ patients reported allergic symptoms, of whom 12 (17.6\%) were diagnosed as having allergy. The physical examination revealed that in addition to deviated septum, 54 patients (79.4\%) had inferior turbinate hypertrophy. In 11 patients (16.2\%), nasal obstruction was attributed also to the middle turbinate ( - Table $\mathbf{1}$ ).

Thirty-eight patients (55.88\%) underwent a CT scan as part of their preoperative surgical assessment. The CT findings revealed significant septal deviation in 37/38 patients (97.4\%), as well as middle meatal abnormalities (mainly obstruction due to concha bullosa) in $18 / 38$ patients (47.4\%). Findings on imaging that are typical of chronic sinusitis, such as sinus opacification, mucosal thickening or OMC obstruction, were found in 22 patients (57.9\%). There was no supporting evidence for these findings in patients' age, gender, medical history, or physical examination (-Table 2 ).

Both patient groups (the group of patients that was sent for imaging and the group that was not) were similar in demographics and clinical findings, with the exception of one main finding; patients who did undergo a CTscan had higher rates of mucosal findings ( mainly hypertrophy and edema) on physical examination ( $4.8 \%$ vs $42.1 \%, p=0.002$ ) ( - Table 1 ).

Of the patients who underwent CTscan, 32 (84.2\%) required a modification of the initial preoperative planning (endoscopic septoplasty and turbinoplasty procedure) based on radiological findings, such as concha bullosa and findings typical of

Table 1 Comparison of anamnestic, preoperative clinical examination and intraoperative findings of the study group

\begin{tabular}{|c|c|c|c|c|}
\hline Variable & Total $(\mathrm{N}=68)$ & No $C T(N=30)$ & $\mathrm{CT}(\mathrm{N}=38)$ & $P$-value \\
\hline Male sex & $50(73.5 \%)$ & $24(80 \%)$ & $26(68.4 \%)$ & 0.28 \\
\hline Female sex & $18(26.5 \%)$ & $6(20 \%)$ & $12(31.6 \%)$ & 0.28 \\
\hline Age & $29.65 \pm 12.12$ [17-69] & $29.90 \pm 13.55$ [18-69] & $29.45 \pm 11.06[17-59]$ & 0.61 \\
\hline Known allergies & $12(17.6 \%)$ & $8(26.7 \%)$ & $4(10.5 \%)$ & 0.08 \\
\hline Snoring & $14(20.6 \%)$ & $5(16.7 \%)$ & $9(23.7 \%)$ & 0.47 \\
\hline \multicolumn{5}{|l|}{ Physical examination } \\
\hline Middle meatus abnormalities & $11(16.2 \%)$ & $0(0 \%)$ & $11(28.9 \%)$ & 0.00 \\
\hline Inferior turbinate hypertrophy & $54(79.4 \%)$ & $24(80 \%)$ & $30(78.9 \%)$ & 0.91 \\
\hline Mucosal edema & $17(28.8 \%)$ & $1(4.8 \%)$ & $16(42.1 \%)$ & 0.00 \\
\hline \multicolumn{5}{|l|}{ Surgery } \\
\hline SMR & $66(97.1 \%)$ & $29(96.7 \%)$ & $37(97.4 \%)$ & $<0.99$ \\
\hline Conchotomy & $62(91.2 \%)$ & $26(86.7 \%)$ & $36(94.7 \%)$ & 0.39 \\
\hline $\begin{array}{l}\text { Surgery extended: ESS/FESS/middle } \\
\text { meatus interference }\end{array}$ & $32(47.1 \%)$ & $0(0 \%)$ & $32(84.2 \%)$ & $>0.00$ \\
\hline
\end{tabular}

Abbreviations: CT, computed tomography; ESS, endoscopic sinus surgery; FESS, functional endoscopic sinus surgery; SMR, submucosal resection of septum. Categorial variables are described as $\mathrm{N}=\%$ (and continuous variables as mean and standard deviation and [] range. 
Table 2 Computed tomography causing a change in surgical planning: comparison of anamnestic, preoperative clinical examination, computed tomography findings and intraoperative findings $(N=38)$

\begin{tabular}{|l|l|l|l|}
\hline Variable & $\begin{array}{l}\text { No change } \\
\text { N=6 }\end{array}$ & $\begin{array}{l}\text { Change } \\
\text { N=32 }\end{array}$ & $P$-value \\
\hline Male sex & $4(66.7 \%)$ & $22(68.8 \%)$ & $>0.99$ \\
\hline Female sex & $2(33.3 \%)$ & $10(31.2 \%)$ & $>0.99$ \\
\hline Age & $24.5 \pm 6.68(21-38)$ & $30.38 \pm 11.54(18-69)$ & 0.46 \\
\hline Known allergies & $0(0 \%)$ & $4(12.5 \%)$ & $<0.99$ \\
\hline Snoring & $1(16.7 \%)$ & $8(25 \%)$ & $<0.99$ \\
\hline Physical examination & $1(16.7 \%)$ & $10(31.3 \%)$ & 0.65 \\
\hline Middle meatus abnormalities & $5(83.3 \%)$ & $25(78.1 \%)$ & $>0.99$ \\
\hline Inferior turbinate hypertrophy & $3(50 \%)$ & $13(40.6 \%)$ & 0.68 \\
\hline Mucosal edema & \multicolumn{2}{|l|}{} \\
\hline CT main findings & $6(100 \%)$ & $28(87.5 \%)$ & $>0.99$ \\
\hline Septal deviation & $2(33.3 \%)$ & $16(50.0 \%)$ & 0.66 \\
\hline Concha bullosa & $2(33.3 \%)$ & $20(62.5 \%)$ & 0.21 \\
\hline Compensatory Inferior turbinate & $1(16.7 \%)$ & $15(45.9 \%)$ & 0.37 \\
\hline Sinus shading, decreased ventilation, OMC narrowing
\end{tabular}

Abbreviation: OMC, osteomeatal complex.

Categorial variables are described as $\mathrm{N}=\%$ (and continuous variables ad mean and standard deviation and [] range.

chronic sinusitis. To identify patients more likely to benefit from imaging studies prior to surgery, those whose operative plans were changed due to CT findings were compared with those whose operative plans remained unchanged. There were no significant differences between the two groups, nor were there any group differences in demographics and clinical findings. Due to the small size of the group of patients who did not benefit from CT scans, we matched the cohort according to gender and age (up to 3 years of age difference). The matching revealed 21 pairs of patients. However, no significant differences were detected in this analysis as well.

Sixty-two patients (91.2\%) underwent septoplasty and inferior turbinate reduction. Additional surgical interventions were performed only among patients that had undergone CT scans that revealed obstructive concha bullosa (requiring resection) or findings suggesting chronic sinusitis (ESS).

To further determine the benefit of CT scanning, a second expert rhinologist, who was blinded to the patients' surgical course, assessed the medical records and CT scans of 20 patients. The assessment was first made without the imaging studies and again with the imaging studies taken under consideration. A change in the preoperative surgical plan was noted in 12 cases (58\%). We defined 3 contribution values $(10 \%$, $20 \%$, and $30 \%$ ) as the minimal threshold values to perform a CT. A comparison of the need for change in the surgical plan (58\%) to the defined values revealed that it was significantly higher than the minimal threshold for statistical significance $(p<0.001, p=0.003$, and $p=0.048$, respectively). Thus, the contribution of CT scans carried a $30 \%(p=0.048)$ chance for a change in the surgical plan from classical SMR and inferior turbinate reduction to a more extended surgical procedure for patients presenting with INO and deviated nasal septum.

\section{Discussion}

Nasal congestion and obstruction are common complaints, and a deviated nasal septum is a common pathology in the rhinology outpatient clinic. The routine methods of assessment of nasal obstruction severity and causes are medical history, anterior rhinoscopy, and flexible fiber optic endoscopy, and their effectiveness is dependent upon physician skills and experience. None of these methods, however, can provide the comprehensive information on the status of the nasal spaces or 3-dimensional understanding of the nasal airflow that is needed for a thorough preoperative evaluation. One large-scale model demonstrated the importance of the physiological airflow of the middle meatus, emphasizing that 50\% of the nasal airflow passes through the middle meatus, while $35 \%$ of it only flows through the inferior meatus. ${ }^{8}$

A CT of the paranasal sinuses is the gold standard imaging for anatomy and pathology of the nasal and paranasal structures. It is not, however, routinely performed for patients presenting with INO. Moreover, the role of the CT scan in the setting of nasal obstruction and septal deviation has not been established in the literature. Several publications failed to demonstrate good correlation between CT findings and the severity of nasal symptoms. Ardeshirpour et al noted that CT findings of septal deviation severity do not necessarily correlate with patient-reported complaints as assessed by the nasal obstruction symptom evaluation (NOSE) scale. ${ }^{9}$ Others observed that CT findings did not always correlate with those related to the nasal septum assessment on physical examination. ${ }^{10}$ On the other hand, Cho et al $^{11}$ reported some correlation between the cross-sectional area of the nasal airway as 
well as the presence of septal deviation on sinus CT scans and patient-reported nasal obstruction symptoms.

The relationship between the appearance of the nasal airflow passage on CT scans in comparison to a thorough physical examination that includes anterior rhinoscopy and nasal fiber optic endoscopy remains unclear. The advantages of preoperative CT imaging before endoscopic sinus surgery are undisputable, to the point that it is considered malpractice to perform sinus surgery without it. ${ }^{7}$ A CT scan is an objective assessment that produces a clear anatomical image that is of utmost importance to the planning and safety of the surgery, as well as being associated with better results. ${ }^{12}$ Yet, when surgery is planned for the alleviation of INO related to septal deviation or other non-inflammatory causes, the role of preoperative CT remains unclear.

The reliability of CT scanning in assessing the nasal septum is undetermined. We observed that CT scans made a major contribution by changing the preoperative plans, and that the

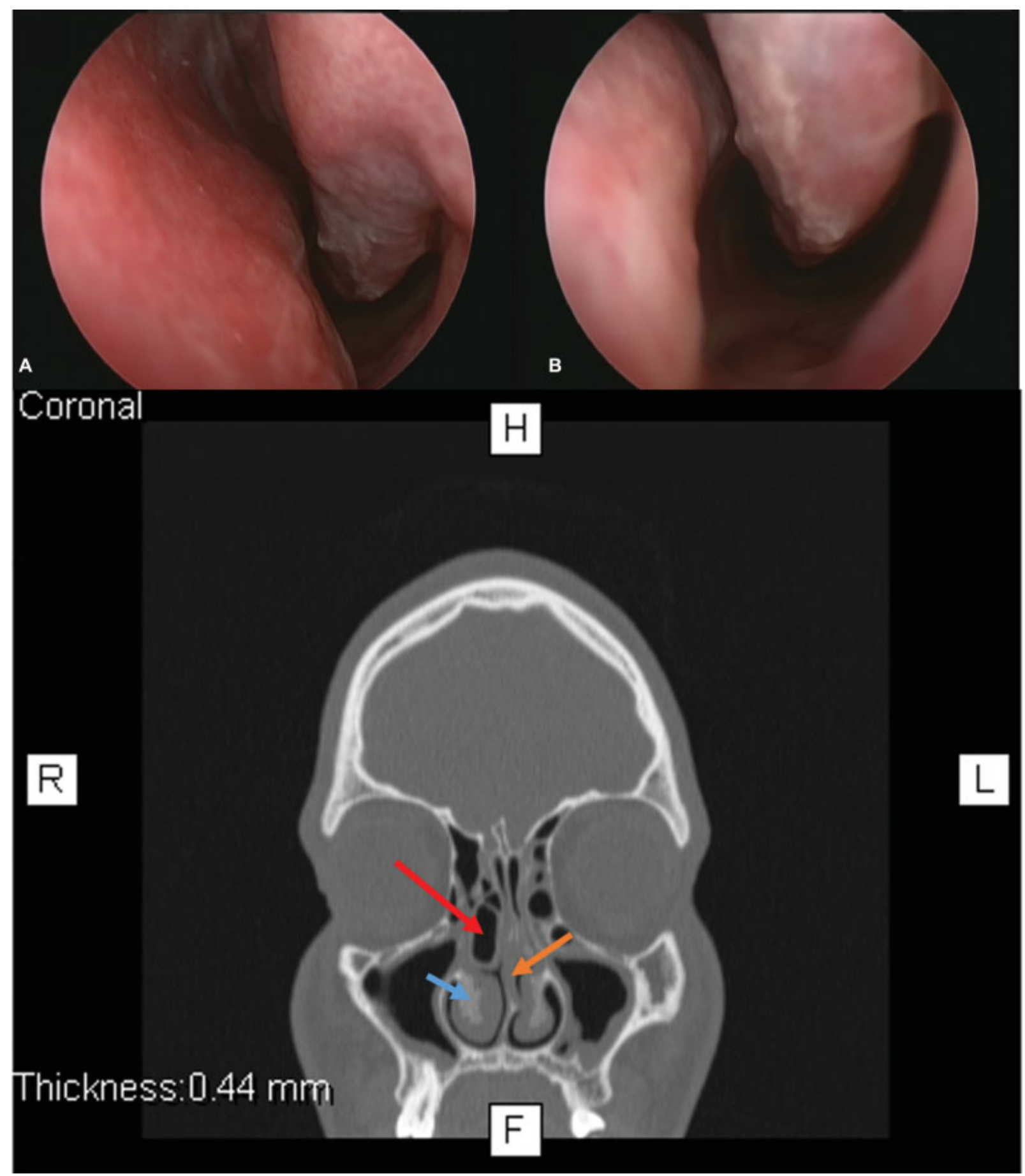

Fig. 2 Representative images of a patient who underwent a computed tomography scan (A) Images of the middle and (B) inferior meatus obtained after application of topical vasoconstrictors and decongestants just prior to surgery. (C) A computed tomography scan demonstrating a very narrow nose due to inferior turbinate hypertrophy (blue arrow) and a severely deviated septum (orange arrow) with high deviation and a concha bullosa (red arrow) that further narrows the passage of air on the right side. 
surgeon subsequently considered anatomical structures other than the septum and inferior turbinate in most of the cases. There was agreement between two expert rhinologists that the CT scan modified the scope of the operation in almost $60 \%$ of cases, and that it contributed to better understanding of the surgical anatomical field in $55 \%$ of cases. Even when there were no inflammatory findings, CT imaging offered better delineation of site of obstruction; for instance, turbinate hypertrophy mainly because of bony thickening, septal thickening and obstructing middle turbinate (-Fig. 2.). In 2013, Karatas et $\mathrm{al}^{5}$ assessed the possible contribution of CT to surgery for nasal obstruction related to septal deviation. Their retrospective study included 76 patients that were assigned to one group of 40 patients who arrived with prior CT scans and 36 patients who had not undergone CT scans beforehand. Their patients were assessed for the detection of pathologies and subjective postoperative relief in nasal airflow. The authors also described a large percentage of patients with concha bullosa (35\%) and chronic sinusitis (17.5\%). Computed tomography was found capable of recognizing pathologic findings that could not be found on physical examination. It was described as being helpful in deciding the location as well as the required type of surgery. Karatas et al found that the additional surgical interventions that were indicated by CT scans that were performed before a planned septoplasty were very helpful in relieving the patient's nasal airway obstruction. However, it is important to highlight that patients with allergic rhinitis were not included in their study, and the authors did not provide their opinion about patients with atopic background and suspected atopic complaints without an established diagnosis of rhinitis.

It should be emphasized that CT is complementary to a thorough clinical assessment among patients with nasal obstruction that may benefit from surgery. We believe that it should be considered especially when obstruction is not anterior to the nasal valve, when it is difficult to assess the middle meatus, and in patients with severe septal deviation that does not allow adequate inspection.

We are aware that CT scans performed in young populations may result in excessive exposure to radiation. The head and neck region is sensitive to radiation, mainly due to accumulation of the radiation in two of the most radiation-sensitive organs encompassed by the scanning field, the thyroid gland and the eye lens, which is at risk for radiation-induced cataract. ${ }^{13}$ There are ongoing attempts to decrease the radiation dose per CT scan, ${ }^{14}$ and the cone beam CT, which requires less radiation, can now serve as an alternative.

As surgical treatment is not free of risks and complications, we believe that optimizing the preoperative evaluation benefits surgical results, thus minimizing risks of further treatments. Moreover, multiple operations may carry additional financial costs.

Our study has several limitations. It is retrospective and includes a relatively small number of patients, which can lead to a biased selection of patients. The main possible bias is that patients were selected to perform a CT scan based on an expert rhinologist clinical judgment. While physical examination is subjective, we believe that the fact that in
$32 / 38$ patients ( $84 \%$ ) an objective assessment with CT imaging was found beneficial overcomes this bias.

We did, however, find significant differences between the two groups of patients and believe that our findings demonstrate several specific advantages of preoperative CT in patients planned for surgery due to nasal obstruction. Our study aimed to investigate the effect of preoperative CT scan on the surgical treatment. The effect on QoL and objective measurement of nasal air flow were not addressed. These are aimed to be further addressed in future prospective studies. Reviewing postoperative follow-up visits, all patients expressed satisfaction from the operation results and none required a revision surgery or additional procedures.

Patients were allocated to CT according to the clinical presentation in our study. Some may suggest that an experienced rhinologist may understand the surgical anatomy only by clinical judgment. However, this subjective examination may also lead to misevaluation, while CT is an objective measurement that gives the most reliable anatomical information relating to the nasal cavity. ${ }^{12}$

\section{Conclusions}

In this series, the initial preoperative planning that was based on physical examination alone was modified in $84.2 \%$ of patients who underwent preoperative imaging for nasal obstruction. Our findings indicate that $\mathrm{CT}$ is a valuable complementary tool for detecting pathological findings that are difficult to detect on physical examination alone. Computed tomography scan may play a pivotal role in assessing septal deviation, especially when the physical examination is limited due to obstruction of one or both nares.

\section{Conflict of Interests}

The authors have no conflict of interests to declare.

\section{References}

1 Tsang CLN, Nguyen T, Sivesind T, Cervin A. Long-term patientrelated outcome measures of septoplasty: a systematic review. Eur Arch Oto-Rhino-Laryngology [Internet]. 2018 May 13 [cited 2019 Jul 27];275(5):1039-48. Available from: http://www.ncbi. nlm.nih.gov/pubmed/29332171

2 Moore M, Eccles R. Objective evidence for the efficacy of surgical management of the deviated septum as a treatment for chronic nasal obstruction: a systematic review. Clin Otolaryngol 2011;36 (02):106-113http://www.ncbi.nlm.nih.gov/pubmed/21332671 [Internet]

3 Stewart MG, Smith TL, Weaver EM, et al. Outcomes after nasal septoplasty: results from the Nasal Obstruction Septoplasty Effectiveness (NOSE) study. Otolaryngol Head Neck Surg 2004; 130(03):283-290http://www.ncbi.nlm.nih.gov/pubmed/ 15054368[Internet]

4 Setzen G, Ferguson BJ, Han JK, et al. Clinical Consensus Statement. Otolaryngol Neck Surg [Internet]. 2012 Nov 10 [cited 2019 Jul 27];147(5):808-16. Available from: http://www.ncbi.nlm.nih.gov/pubmed/23054429

5 Karataş D, Yüksel F, Şentürk M, Doğan M. The contribution of computed tomography to nasal septoplasty. J Craniofac Surg 2013;24(05):1549-1551http://www.ncbi.nlm.nih.gov/pubmed/ 24036724[Internet] 
6 Shpilberg KA, Daniel SC, Doshi AH, Lawson W, Som PM. CT of Anatomic Variants of the Paranasal Sinuses and Nasal Cavity: Poor Correlation With Radiologically Significant Rhinosinusitis but Importance in Surgical Planning. AJR Am J Roentgenol 2015; 204(06):1255-1260http://www.ajronline.org/doi/10.2214/ AJR.14.13762[Internet]

7 Rosenfeld RM, Piccirillo JF, Chandrasekhar SS, et al. Clinical Practice Guideline (Update): Adult Sinusitis. Otolaryngol Neck Surg 2015 Apr [cited 2019 Jul 27]152(02):S1-39. Available from: http://www.ncbi.nlm.nih.gov/pubmed/25832968

8 Scherer PW, Hahn II, Mozell MM. The biophysics of nasal airflow. Otolaryngol Clin North Am 1989;22(02):265-278http://www. ncbi.nlm.nih.gov/pubmed/2748174[Internet]

9 Ardeshirpour F, Mccarn KE, Mckinney AM, Odland RM, Yueh B, Hilger PA. Computed Tomography Scan Does Not Correlate With Patient Experience of Nasal Obstruction. 2016:820-825

10 Sedaghat AR, Kieff DA, Bergmark RW, Cunnane ME, Busaba NY. Radiographic evaluation of nasal septal deviation from computed tomography correlates poorly with physical exam findings. Int Forum Allergy Rhinol 2015;5(03):258-262http://www.ncbi.nlm. nih.gov/pubmed/25412986[Internet]
11 Cho GS, Kim JH, Jang YJ. Correlation of nasal obstruction with nasal cross-sectional area measured by computed tomography in patients with nasal septal deviation. Ann Otol Rhinol Laryngol 2012;121(04):239-245http://www.ncbi.nlm.nih.gov/pubmed/ 22606927[Internet]

12 Cashman EC, Macmahon PJ, Smyth D. Computed tomography scans of paranasal sinuses before functional endoscopic sinus surgery. World J Radiol 2011;3(08):199-204http://www.ncbi. nlm.nih.gov/pubmed/22022638[Internet]

13 Maruyama T, Nishizawa K, Kumamoto Y, Noda Y, Iwai K, Furuya Y. [Estimation of frequency, population doses and stochastic risks in brachytherapy in Japan, 1983]. Nihon Igaku Hoshasen Gakkai Zasshi [Internet]. 1988 May 25 [cited 2019 Jul 27];48 (5):633-40. Available from: http://www.ncbi.nlm.nih.gov/ pubmed/3412876

14 Brem MH, Zamani AA, Riva R, et al. Multidetector CT of the paranasal sinus: potential for radiation dose reduction. Radiology 2007;243(03):847-852http://www.ncbi.nlm.nih.gov/pubmed/ 17517938 [Internet] 